Open Access

\title{
Cross-calibration of the Siemens mMR: easily acquired accurate PET phantom measurements, long-term stability and reproducibility
}

Sune H. Keller ${ }^{1 *}$, Björn Jakoby ${ }^{2,3}$, Susanne Svalling ${ }^{1}$, Andreas Kjaer ${ }^{1}$, Liselotte Højgaard ${ }^{1}$ and Thomas L. Klausen ${ }^{1}$

\footnotetext{
* Correspondence: sune@pet.rh.dk '3982 Department of Clinical Physiology, Nuclear Medicine and PET, Rigshospitalet (University of Copenhagen), Blegdamsvej 9, DK-2100 Copenhagen, Denmark Full list of author information is available at the end of the article
}

\begin{abstract}
Background: We present a quick and easy method to perform quantitatively accurate PET scans of typical water-filled PET plastic shell phantoms on the Siemens Biograph mMR PET/MR system.

We perform regular cross-calibrations (Xcal) of our PET systems, including the PET/MR, using a Siemens $\mathrm{mCT}$ water phantom.

Long-term stability: The mMR calibration stability was evaluated over a 3-year period where 54 cross-calibrations were acquired, showing that the mMR on average underestimated the concentration by $16 \%$, consistently due to the use of MR-based $\mu$-maps.

The mMR produced the narrowest calibration ratio range with the lowest standard deviation, implying it is the most stable of the six systems in the study over a 3-year period.

mMR accuracy with predefined $\boldsymbol{\mu}$-maps: With the latest mMR software version, VB20P, it is possible to utilize predefined phantom $\mu$-maps. We evaluated both the system-integrated, predefined $\mu$-map of the long mMR water phantom and our own user-defined CT-based $\mu$-map of the $\mathrm{mCT}$ water phantom, which is used for cross-calibration.

For seven scans, which were reconstructed with correctly segmented $\mu$-maps, the $\mathrm{mMR}$ produced cross-calibration ratios of 1.00-1.02, well within the acceptance range [0.95-1.05], showing high accuracy.
\end{abstract}

Conclusions: The mMR is the most stable PET system in this study, and the mean underestimation is no longer an issue with the easily accessible $\mu$-map, which resulted in correct cross-calibration ratios in all seven tests. We will share the user-defined $\mu$-map of the $\mathrm{mCT}$ phantom and the protocol with interested mMR users.

Keywords: PET/MR, Quality control, Cross-calibration, Calibration, PET phantom 


\section{Findings}

\section{Introduction}

Accurate PET measurements on PET/MR systems are problematic with MR-based attenuation correction (MRAC) [9, 11, 12]. For phantoms, the plastic materials in MR-based $\mu$-maps are typically segmented as air and are assigned with a linear attenuation coefficient (LAC) of $0 \mathrm{~cm}^{-1}$ when they ought to have LACs close to that of water $[2,11,12]$.

For single phantom scans, one can utilize externally acquired, co-registered, and converted (Hounsfield units (HU) to $511 \mathrm{keV}$ LACs) CT-based $\mu$-maps [8] or calculated $\mu$-maps [4]. This approach is time-consuming and error-prone, and it requires either the use of external $\mu$-maps or access to an external reconstruction setup. Thus, it is not a suitable approach for regularly repeated phantom scans, e.g., for routine quality control (QC) such as our regularly performed crosscalibrations (Xcal) $[1,6]$.

Cross-calibration is a quality control procedure to check the quantitative accuracy of our systems (PET, PET/CT, and PET/MR) and other equipment (Fig. 1).

In the current work, we firstly present a quick and easy method to perform quantitatively accurate PET scans of a typical water-filled plastic shell cylinder phantom on the Siemens mMR PET/MR system. We describe how to integrate an external, CT-based $\mu$-map into the system software as a user-defined $\mu$-map for routine use.

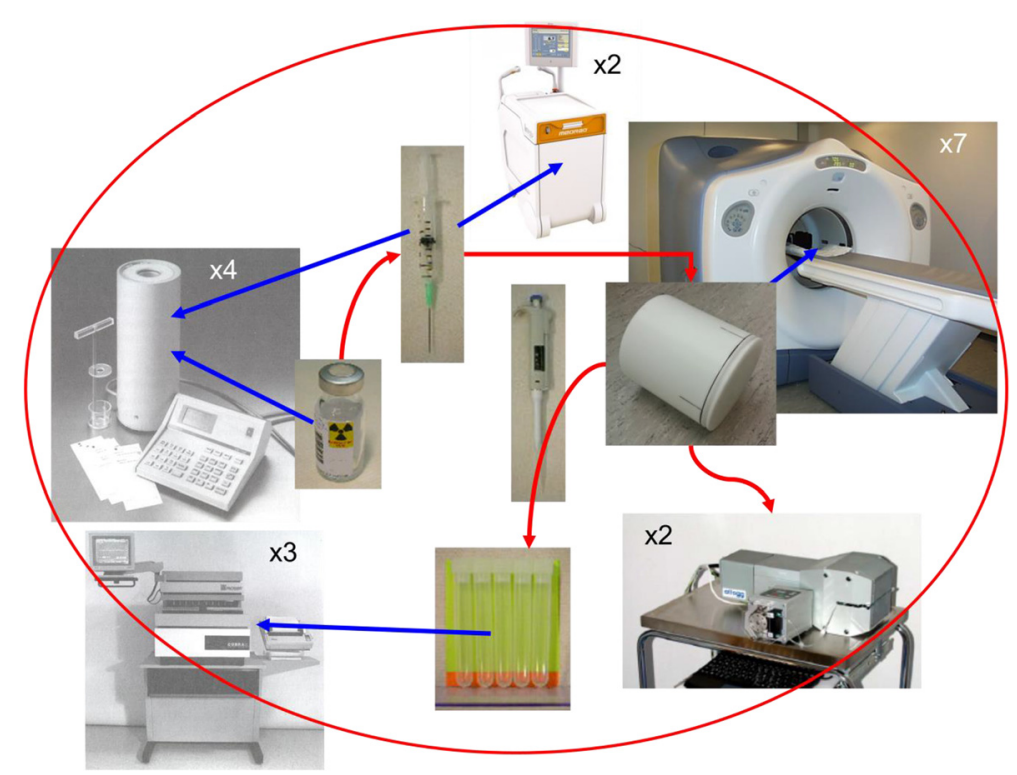

Fig. 1 Cross-calibration procedure. To ensure quantitatively accurate measurements of activities at our PET center, we perform a quality control of our devices every 2-3 weeks, testing they are calibrated to measure within our acceptance range. Since this quality control (QC) procedure cross-checks the calibration of all devices compared to a reference device, a Veenstra VDC-404 dose calibrator, it is denoted a cross-calibration $[1,6]$. We include all devices in use, which as of 2015 are as follows: one PET/MR, five PET/CT systems (one is exclude in our 3-year comparison as it has only been installed for 2 of the 3 years), one HRRT PET system, three well counters, two blood samplers, two automatic injectors, and four dose calibrators (incl. the reference). Each device measures the original dose, the filled phantom, or samples drawn from the phantom as illustrated above 
As a second part of the assessment of the mMR phantom scan performance, we evaluate the results of executing cross-calibrations every 2-3 weeks over a 3-year period, comparing the mMR to five other PET systems to assess the long-term stability and reproducibility.

\section{Material and methods}

The PET/MR system under evaluation is the Siemens Biograph mMR [2, 4] at Rigshospitalet, Copenhagen. The cross-calibration phantom we used in this study was a Siemens mCT water phantom (Fig. 2a), for which we had acquired a CT scan on a Siemens Biograph mCT PET/CT system (Fig. 2b) [7].

All PET scans on the mMR were acquired for $5 \mathrm{~min}$ with the phantom placed in the lowest vertical position on the mMR phantom holder and positioned axially using the positioning laser and scanning with a fixed protocol to ensure we always scanned in the same position. The $\mathrm{mCT}$ phantom was scanned with varying concentrations of $\left[{ }^{18} \mathrm{~F}\right]$-FDG in water (see Table 1 ).

The mMR PET images were reconstructed on the mMR using OP-OSEM with 4 iterations, 21 subsets, and a 3-mm FWHM Gaussian post-reconstruction filter into $344 \times 344 \times 127$ matrices of $0.83 \times 0.83 \times 2.03 \mathrm{~mm}^{3}$ voxels.

We used a Veenstra VDC 404 dose calibrator as reference, and all devices in the cross-calibration should measure the same concentration within $\pm 5 \%$ of the reference concentration, i.e., have Xcal concentration ratios in the range [0.95-1.05].

\section{Predefined $\mu$-map options as alternatives to the standard Dixon MR-based $\mu$-maps}

The mMR software (version VB20P, available since Q4 2013) offers the choice of four easily accessible predefined $\mu$-maps for phantom reconstructions as alternative to the standard Dixon MR-based $\mu$-maps. Two of the predefined choices are user-defined options, which we use to test our external CT-based $\mu$-map. As an alternative, we also tested the $\mu$-map of the mMR water phantom, which is given as one of the two predefined and build-in $\mu$-maps (Fig. 2c): The $\mathrm{mCT}$ and $\mathrm{mMR}$ water phantoms are made of the same material and have the same diameter and wall thickness (see Fig. 2). We acquired and reconstructed seven PET scans on the mMR of the $\mathrm{mCT}$ phantom using
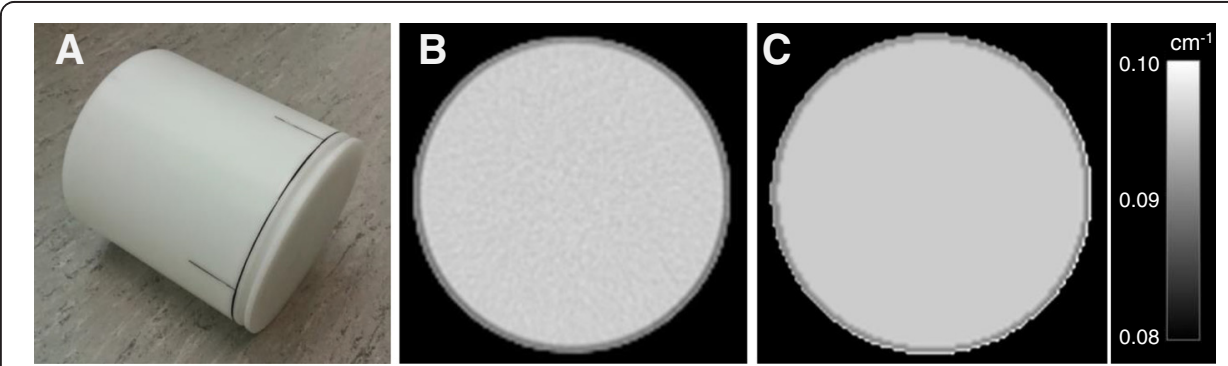

Fig. 2 The phantom used for cross-calibration scans. a The Siemens $\mathrm{mCT}$ water phantom of $20 \mathrm{~cm}$ inner diameter and axial length, $21 \mathrm{~cm}$ outer diameter, and $6283 \mathrm{ml}$ volume. $\mathbf{b}$ CT scan of the phantom after conversion to linear attenuation coefficient map ( $\mu$-map). c The calculated $\mu$-map of the water phantom supplied with the mMR. We can use the $\mu$-map in c directly as we scan the $\mathrm{mCT}$ water phantom in the default position of the mMR water phantom, which is made of the same material and has the same inner and outer diameters as the $\mathrm{mCT}$ phantom in a but is $10 \mathrm{~cm}$ longer to cover the longer PET FOV of the mMR. The $\mu$-map in $\mathbf{c}$ is given as a predefined $\mu$-map in the mMR system reconstruction software (version VB2OP) 
Table 1 Test of $\mu$-maps for mMR cross-calibration: concentrations $(\mathrm{kBq} / \mathrm{ml})$ and ratios

\begin{tabular}{llll}
\hline$\mu$-map & Reference concentration & mMR concentration & mMR Xcal concentration ratio \\
\hline CT-based (+8 mm in $x$ ) & 10.77 & 10.79 & 1.00 \\
CT-based (-10 mm in $x)$ & 10.77 & 10.79 & 1.00 \\
CT-based (-2 mm in $x)$ & 18.78 & 19.04 & 1.01 \\
CT-based (no shift) & 11.29 & 11.57 & 1.02 \\
mMR water (original) & 11.86 & 11.96 & 1.01 \\
mMR water (original) & 10.77 & 10.91 & 1.01 \\
mMR water (original) & 11.29 & 11.55 & 1.02 \\
\hline
\end{tabular}

five different predefined $\mu$-maps. In three cases, the $\mu$-maps were shifted up to $10 \mathrm{~mm}$ in the $x-y$ plane testing robustness against misregistrations.

\section{Creation of a user-defined CT-based $\mu$-map}

After a $120-\mathrm{kVp}$ CT scan of the $\mathrm{mCT}$ phantom, the CT image was automatically registered to an mMR PET scan of the mCT phantom using Vinci 2.55 [10]. HU were converted to linear attenuation coefficients at $511 \mathrm{keV}$ in Matlab following the method of Carney et al. [3], where $\mathrm{LAC}=9.6 \times 10^{5} \times(\mathrm{HU}+1000)$. A $3-\mathrm{mm}$ filter was applied in Vinci, and images were saved in interfile format. Header text files were then generated according to the specifications in [5]. The registration procedure above served mostly to correct any rotational differences because the translational positioning of the $\mu$-map had to be set in the header, specifying a $\mu$-map origin in pixels and an origin offset in millimeters relative to the system patient table origin. This requires fixed phantom positioning for all PET scans performed using this $\mu$-map. Finally, the image and the corresponding header were named User_Defined_n.v(.hdr) $(n=1,2)$ and saved in a dedicated folder for predefined $\mu$-maps.

\section{Routine cross-calibration}

The mMR has been included in our routine cross-calibration procedure since its installation but has not yielded concentration ratios in the $\pm 5 \%$ acceptance range while using MR-based $\mu$-maps, which segments plastic as air. Even though the mMR PET images using MR-based $\mu$-maps are incorrectly quantified, they are consistently (and reproducibly) erroneous, meaning we can still use the cross-calibration results to evaluate the stability of the system in comparison with our five other PET systems (details in Table 2) included in the cross-calibration during the same 3-year period (02/2012-01/ 2015). Since the mMR has been calibrated three times using a correct $\mu$-map (and a complex procedure) in the 3 -year period, we are certain that it measures accurately and the consistent bias is purely due to the incorrect MR-based $\mu$-maps.

Table 2 Statistics of the Xcal ratios for six systems over 3 years

\begin{tabular}{llll}
\hline System & Mean & SD & Range [min-max] \\
\hline PET1: Siemens HRRT & 0.99 & 0.0269 & {$[0.94-1.05]$} \\
PET3: Siemens Biograph mCT & 1.00 & 0.0266 & {$[0.95-1.04]$} \\
PET4: Siemens Biograph mCT & 1.01 & 0.0265 & {$[0.95-1.06]$} \\
PET5: Siemens Biograph True-Point TrueV & 1.01 & 0.0238 & {$[0.95-1.06]$} \\
PET6: Siemens Biograph True-Point TrueV & 1.00 & 0.0209 & {$[0.95-1.04]$} \\
PET7: Siemens Biograph mMR & 0.84 & 0.0143 & {$[0.81-0.88]$} \\
\hline
\end{tabular}


All measurements of the concentrations in the images were performed in Siemens TrueD using a cylindrical volume of interest (VOI) placed centrally in the phantoms, $12-15 \mathrm{~cm}$ in diameter and $500-950 \mathrm{~cm}^{3}$ in volume.

Results

Test of $\mu$-maps

We acquired and reconstructed seven PET scans on the mMR of the mCT phantom using five different $\mu$-maps (Table 1). All images resulted in Xcal ratios well within the acceptance range [0.95-1.05] as reported in Table 1. Examples of fused images are shown in Fig. 3.

As it took a few cycles to get the translational position of the user-defined $\mu$-map set correctly in the image header, we found that obtaining the Xcal ratios in the center of the phantom is robust against misregistrations of up to $10 \mathrm{~mm}$ on the $x$-axis (results in Table 1). Plotting horizontal profiles through the image slices showed a clear gradient across the $\mathrm{mCT}$ phantom in the $x$-direction when using the $\mu$-maps shifted by 8 and $10 \mathrm{~mm}$ as seen in Fig. 4. Therefore, only cylindrical VOIs placed balanced around the center guarantees valid measurements with these shift magnitudes. When using the 2 -mm shifted $\mu$-map or the original mMR water phantom $\mu$-map with a $1-2-m m$ shift in the $x-y$ plane (Fig. 3b), a very weak, negligible gradient is observed. Although, the predefined $\mu$-map of the mMR water phantom is longer and thus matches a $z$-position, which is different than that of the shorter $\mathrm{mCT}$ phantom, it can still be used as a $\mu$-map for the $\mathrm{mCT}$ phantom; VOIs in the central parts of the reconstructed PET images are not affected.

\section{Long-term $m M R$ calibration stability}

We performed 59 cross-calibrations over a 3-year period, with the mMR left out five times: once, the system was unavailable, and at four occasions, the MR-based $\mu$-map was accidentally reconstructed (irreversibly) without the two-compartment phantom segmentation option selected, yielding a $\mu$-map with the water segmented as fat ( $\mathrm{LAC}=0.085 \mathrm{~cm}^{-1}$ instead of $0.096 \mathrm{~cm}^{-1}$ ) causing Xcal ratios at around 0.70 . Figure 5 shows all included measurements on the six systems over the 3-year period.

Table 2 shows that the mMR measures activity concentrations are $16 \%$ too low on average due to the use of MR-based $\mu$-maps with the plastic body of the phantom segmented as air. All other systems measure very close to 1.00 on average.

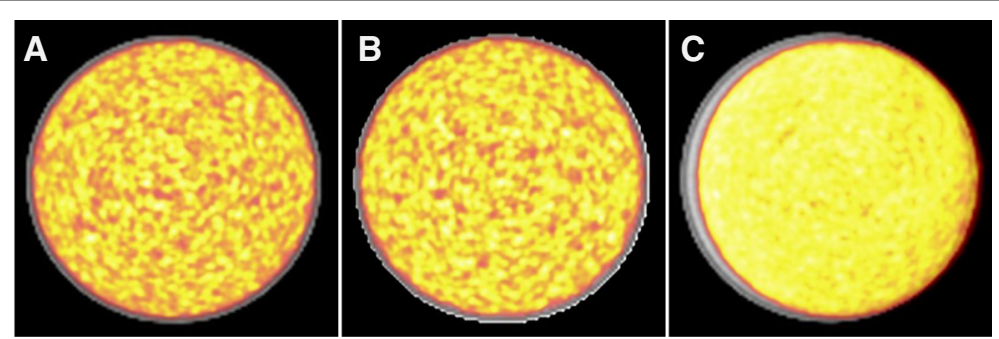

Fig. 3 Fusion of $\mu$-maps and mMR cross-calibration PET scans of the mCT phantom. a User-defined CT-based (no shift) $\mu$-map. b Predefined original mMR water phantom $\mu$-map. c CT-based $\mu$-map with an 8-mm shift (misregistration) 


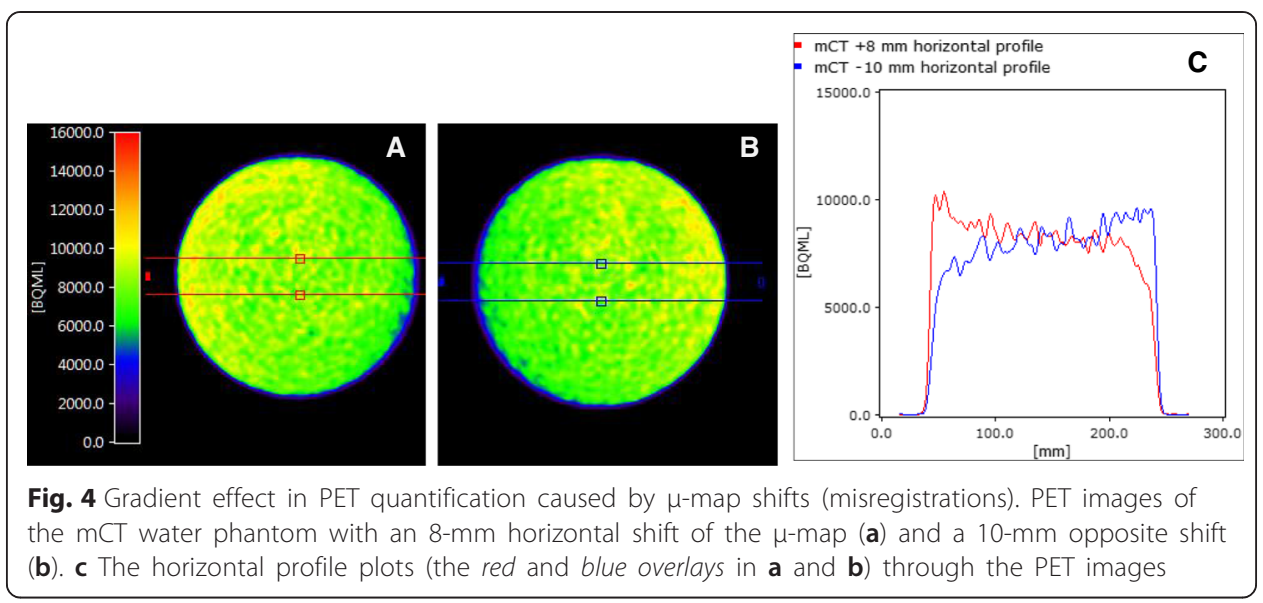

We only had ratios out of the acceptance range in 5 out of 290 scans (PET1: $2 \times 0.94$, PET4: $1 \times 1.06$, and PET5: $2 \times 1.06$ ) and newer twice in a row (see Fig. 5).

\section{Discussion}

The results in Table 2 show that the mMR has a smaller standard deviation (SD) and narrower range of cross-calibration ratios than the other systems, which implies high reproducibility. Scaling the $54 \mathrm{mMR}$ Xcal ratios reconstructed with the MR-based $\mu$-map to a mean of 1.00 by dividing each by the actual mean (0.84) changes the range to [0.97-1.05], which is still narrower than for any other systems in this study, and the SD changes to 0.0170 (still the lowest). Thus, the mMR is the most stable of the systems over a 3-year period, which could be caused by its use of avalanche photodiode (APD) PET detectors instead of conventional photomultiplier tubes (PMTs).

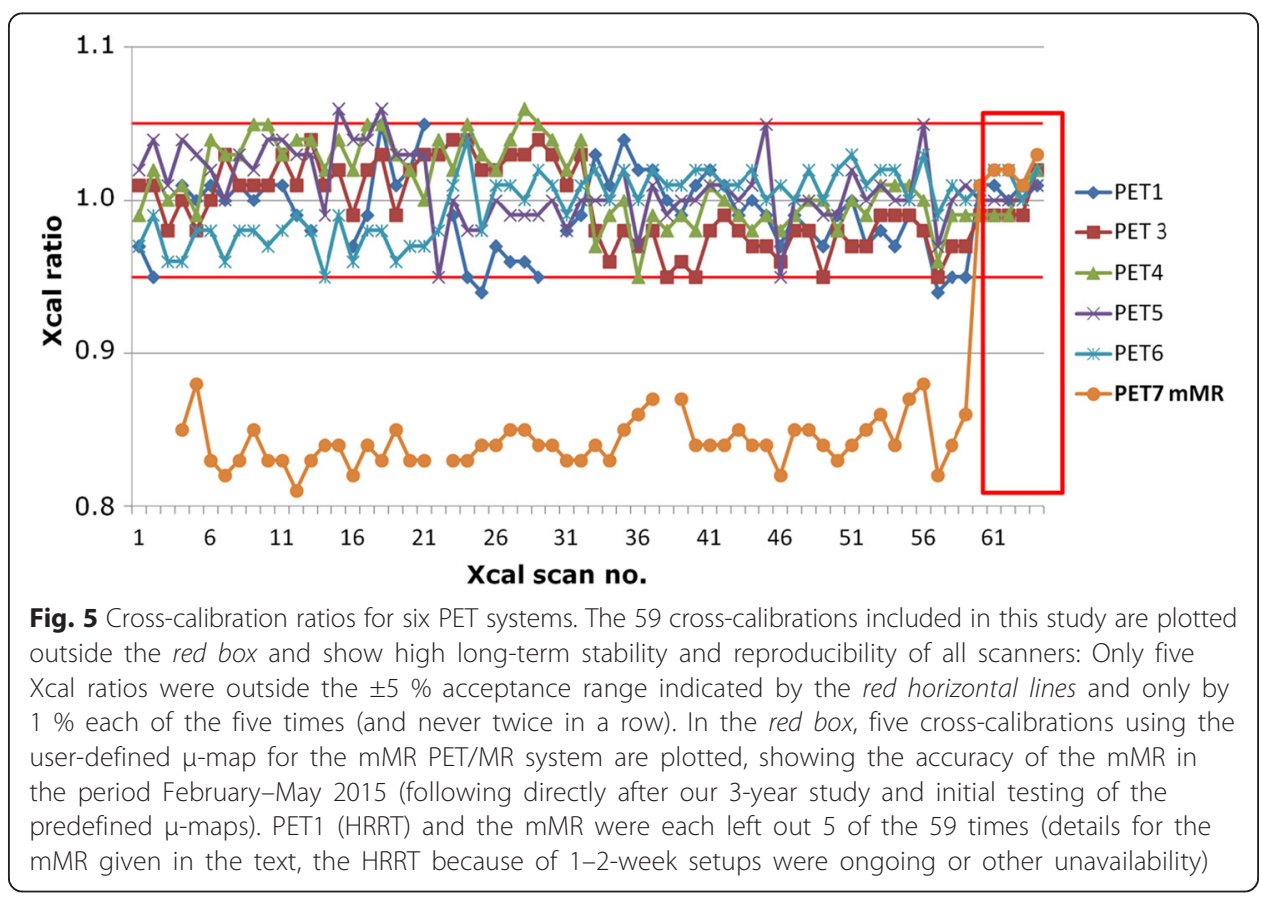


The long-term stability and accuracy of all six PET systems in this study were high with only 5/290 measures out of range (off by $1 \%$ each time and newer off twice in a row) warranting no further actions to ensure the systems measure accurately. The correction would be to redetermine the ECAT calibration factors (ECFs) normally only adjusted when a new $\left[{ }^{68} \mathrm{Ge}\right]$-phantom for daily QC, normalization, and setup is put in use (at 1.5-year intervals).

This phantom study is limited to cross-calibrations using $\left[{ }^{18} \mathrm{~F}\right]$-FDG. But the $\mu$-maps used are tracer-independent, and similar $\mu$-maps could be generated for other phantoms scanned on a regular basis in a fixed position.

\section{Conclusion}

Over a 3-year period and 54 cross-calibrations, the mMR showed to be the most stable of the six PET systems evaluated in this study. The Xcal ratios were persistently off by a factor of $16 \%$ due to the use of MR-based $\mu$-maps, a factor that we can now easily eliminate by using correct $\mu$-maps.

We have successfully demonstrated a procedure to perform accurate crosscalibration of the mMR PET/MR system. Both a new CT-based user-defined $\mu$-map of the $\mathrm{mCT}$ water phantom and a predefined $\mu$-map of the $\mathrm{mMR}$ water phantom resulted in accurate cross-calibration ratios. The $\mu$-maps are available as an easily accessible drop-down option in the system's user interface.

We will share our user-defined $\mu$-map of the $\mathrm{mCT}$ phantom and the protocol with interested mMR users, who wish to employ our method. Following our work, one can also generate user-defined $\mu$-maps for other frequently used phantoms. If compliant with local procedures, the mMR water phantom can also be used across systems for cross-calibrations.

Competing interests

Björn Jakoby is employed by Siemens Healthcare $\mathrm{GmbH}$. The authors declare that they have no competing interests.

\section{Authors' contributions}

SK, SS, and TK designed the study, managed the data, carried out the reconstructions and measurements, and drafted the manuscript. BJ developed the method for build-in user- and predefined $\mu$-maps and revised the manuscript. AK and LH participated in the interpretation of the data and revised the manuscript critically. All authors read and approved the final manuscript.

Acknowledgements

We kindly thank The John and Birthe Meyer Foundation who donated the mMR PET/MR system to Rigshospitalet.

\section{Author details}

13982 Department of Clinical Physiology, Nuclear Medicine and PET, Rigshospitalet (University of Copenhagen), Blegdamsvej 9, DK-2100 Copenhagen, Denmark. ²Diagnostic Imaging, Magnetic Resonance, Siemens Healthcare $\mathrm{GmbH}$, Allee am Roethelheimpark 2, 91052 Erlangen, Germany. ${ }^{3}$ University of Surrey, Guildford, UK.

Received: 7 April 2016 Accepted: 8 June 2016

Published online: 07 July 2016

\section{References}

1. Boellaard R, Delgado-Bolton R, Oyen WJ, Giammarile F, Tatsch K, Eschner W, et al. FDG PET/CT: EANM procedure guidelines for tumour PET imaging: version 2.0. Eur J Nucl Med Mol Imaging. 2015;42:328-54.

2. Boellaard R, Rausch I, Beyer T, Delso G, Yaqub M, Quick HH, et al. Quality control for quantitative multicenter whole-body PET/MR studies: a NEMA image quality phantom study with three current PET/MR systems. Med Phys. 2015:42:5961-9.

3. Carney JP, Townsend DW, Rappoport V, Bendriem B. Method for transforming CT images for attenuation correction in PET/CT imaging. Med Phys. 2006;33:976-83.

4. Delso G, Fürst S, Jakoby B, Ladebeck R, Ganter C, Nekolla SG, et al. Performance measurements of the Siemens mMR integrated whole-body PET/MR scanner. J Nucl Med. 2011;52:1-9.

5. Fenchel M. Support of customer defined hardware $\mu$-maps, vol. 01. Siemens: Whitepaper; 2011. 
6. Geworski L, Knoop BO, de Wit M, Ivancevic V, Bares R, Munz DL. Multicenter comparison of calibration and cross calibration of PET scanners. J Nucl Med. 2002:43:635-9.

7. Jakoby BW, Bercier Y, Conti M, Casey ME, Bendriem B, Townsend DW. Physical and clinical performance of the mCT time-of-flight PET/CT scanner. Phys Med Biol. 2011;56:2375-89.

8. Keereman V, Mollet P, Fierens Y, Espana S, Vandenberghe S. Design of a realistic PET-CT-MRI phantom. IEEE Nucl Sci Symp Conf Rec. 2011;3173-7.

9. Keller SH, Hansen AE, Holm S, Beyer T. Image distortions in clinical PET/MR imaging. In: Carrio I, Ros P, editors. PET/MRI. Heidelberg: Springer; 2014. p. 21-41.

10. Max-Planck Institut für Neurologische Forschung. Vinci Online Resources. 2012. http://www.nf.mpg.de/vinci/ index2.html. Accessed 21 June 2016.

11. Oprea-Lager DE, Yaqub M, Pieters IC, Reinhard R, van Moorselaar RJA, van den Eertwegh AJM, et al. A clinical and experimental comparison of time of flight PET/MRI and PET/CT systems. Mol Imaging Biol. 2015;17:714-25.

12. Ziegler S, Braun H, Ritt P, Hocke C, Kuwert T, Quick HH. Systematic evaluation of phantom fluids for simultaneous PET/MR hybrid imaging. J Nucl Med. 2013;54:1464-71.

Submit your manuscript to a SpringerOpen ${ }^{\circ}$ journal and benefit from:

- Convenient online submission

- Rigorous peer review

- Immediate publication on acceptance

- Open access: articles freely available online

- High visibility within the field

- Retaining the copyright to your article

Submit your next manuscript at $>$ springeropen.com 